\title{
Graded activation of CRAC channel by binding of different numbers of STIM1 to Orai1 subunits
}

\author{
Zhengzheng $\mathrm{Li}^{1,{ }^{*}}$, Lin $\mathrm{Liu}^{2,}{ }^{*}$, Yongqiang Deng ${ }^{1,{ }^{*}}$, Wei $\mathrm{Ji}^{1}$, Wen $\mathrm{Du}^{1}$, Pingyong $\mathrm{Xu}^{1}$, Liangyi Chen ${ }^{1}$, Tao $\mathrm{Xu}^{1,2}$ \\ ${ }^{I}$ National Key Laboratory of Biomacromolecules, Institute of Biophysics, Chinese Academy of Sciences, Beijing 100101, China; \\ ${ }^{2}$ College of Life Science and Technology, Huazhong University of Science and Technology, Wuhan 430074, China
}

The $\mathrm{Ca}^{2+}$ release-activated $\mathrm{Ca}^{2+}$ (CRAC) channel pore is formed by Orai1 and gated by STIM1 after intracellular $\mathrm{Ca}^{2+}$ store depletion. To resolve how many STIM1 molecules are required to open a CRAC channel, we fused different numbers of Orai1 subunits with functional two-tandem cytoplasmic domains of STIM1 (residues 336-485, designated as $\mathbf{S}$ domain). Whole-cell patch clamp recordings of these chimeric molecules revealed that CRAC current reached maximum at a stoichiometry of four Orail and eight $S$ domains. Further experiments indicate that two-tandem S domains specifically interact with the C-terminus of one Orai1 subunit, and CRAC current can be gradually increased as more Orai1 subunits can interact with $S$ domains or STIM1 proteins. Our data suggest that maximal opening of one CRAC channel requires eight STIM1 molecules, and support a model that the CRAC channel activation is not in an "all-or-none" fashion but undergoes a graded process via binding of different numbers of STIM1.

Keywords: CRAC channel; Orai1; STIM1; calcium store; stoichiometry

Cell Research (2011) 21: 305-315. doi:10.1038/cr.2010.131; published online 14 September 2010

\section{Introduction}

Activation of cell surface receptors that activate phospholipase $\mathrm{C}$ causes $\mathrm{Ca}^{2+}$ release from the endoplasmic reticulum (ER) $\mathrm{Ca}^{2+}$ store. The resulting depletion of intracellular $\mathrm{Ca}^{2+}$ store induces extracellular $\mathrm{Ca}^{2+}$ influx through CRAC channels [1, 2]. STIM1 [3, 4] and Orai1 [5-7] are essential proteins of the CRAC channel. STIM1 is localized at the ER and functions as the sensor of ER luminal $\mathrm{Ca}^{2+}$ concentration $[3,8]$, and Orail is the channel pore subunit at the plasma membrane (PM) [9-11]. STIM1 aggregates underneath the PM after store depletion [3, 12-14] and activates the CRAC channel by directly binding to Orai1 $[15,16]$.

Knowledge of the stoichiometry of the CRAC channel is crucial to understanding the mechanisms of channel assembly, gating and function. We and others have previously demonstrated that Orail forms tetramers in the

\footnotetext{
*These three authors contributed equally to this work.

Correspondence: Tao $\mathrm{Xu}^{\mathrm{a}}$, Liangyi Chen ${ }^{\mathrm{b}}$

${ }^{a}$ E-mail: xutao@ibp.ac.cn

bE-mail: chen_liangyi@yahoo.com

Received 21 July 2010; revised 9 August 2010; accepted 11 August 2010; published online 14 September 2010
}

PM [17-20]. We have also demonstrated that one CRAC channel contains at least two STIM1 full-length cytosolic fragments (CT-STIM1) [17-20], which suggests that a STIM1 dimer may be enough to open the CRAC channel. This result seems to disagree with the general belief that the oligomerization of full-length STIM1 is required for the channel activation [21, 22]. It should be pointed out that in the previous paper [17], we deliberately expressed STIM1 at very low levels to ensure the detection of single molecule fluorescence, and did not document whether there was full activation of Orail channels at those low levels of CT-STIM1 expression. Thus, it remains unclear whether the Orail activated by CT-STIM1 and the Orail activated by full-length STIM1 after store depletion are of the same stoichiometry. Therefore, how many STIM1 molecules are required to fully activate a CRAC channel remains an open question. Recently, small cytosolic fragments of STIM1, such as SOAR (residues 344-442) [23] and CAD (residues 342-448) [15] have been identified as core domains of STIM1 that efficiently activate Orail channels without depletion of ER $\mathrm{Ca}^{2+}$ stores. These functional fragments provide us a substitution of the full-length STIM1 in activating CRAC channel. Inspired by the approach that utilized fusing chimeras of the L-type $\mathrm{Ca}^{2+}$ channel and calmodulin to 
investigate the stoichiometry of channel/calmodulin interaction in $\mathrm{Ca}^{2+}$-dependent channel inactivation [24], we linked Orai1 with different numbers of functional STIM1 domain to investigate the STIM1/Orail stoichiometry in the activation of the CRAC channel.

\section{Results}

Orail linked with cytosolic STIM1 fragments produces constitutive CRAC-like current

We chose functional cytosolic STIM1 fragments with less tendency to aggregate to avoid excessive interactions between STIM1 fragments that might affect the assembly and transport of the linked Orail subunits. An initial attempt using SOAR domain was not successful, because the SOAR-linked Orail tended to form aggregates in the ER without being transported to the PM. Most functional cytosolic STIM1 fragments purified in vitro form dimmers [16, 23], and CAD forms tetramers [15]. Hence, we next selected the dimeric STIM1 fragment (residues 336-485), termed as S domain here, which activates the Orail channel efficiently [23]. First, tandem S domains (SS) were constructed by linking two $\mathrm{S}$ domains with a 24-amino acid linker sequence (L1 in Figure 1A; see Methods and Supplementary information, Figure S1 for constructs used in this study). Coexpressing Orail with dimeric SS domains generated constitutive CRAC-like currents in HEK293 cells, whereas no observable current

A

B

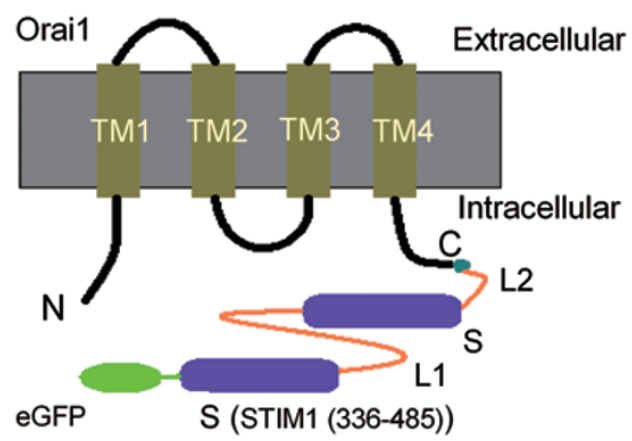

L1: GGSGGSGGGILQSRGGSGGSGGSG

L2: TSRGYQATMGGSG
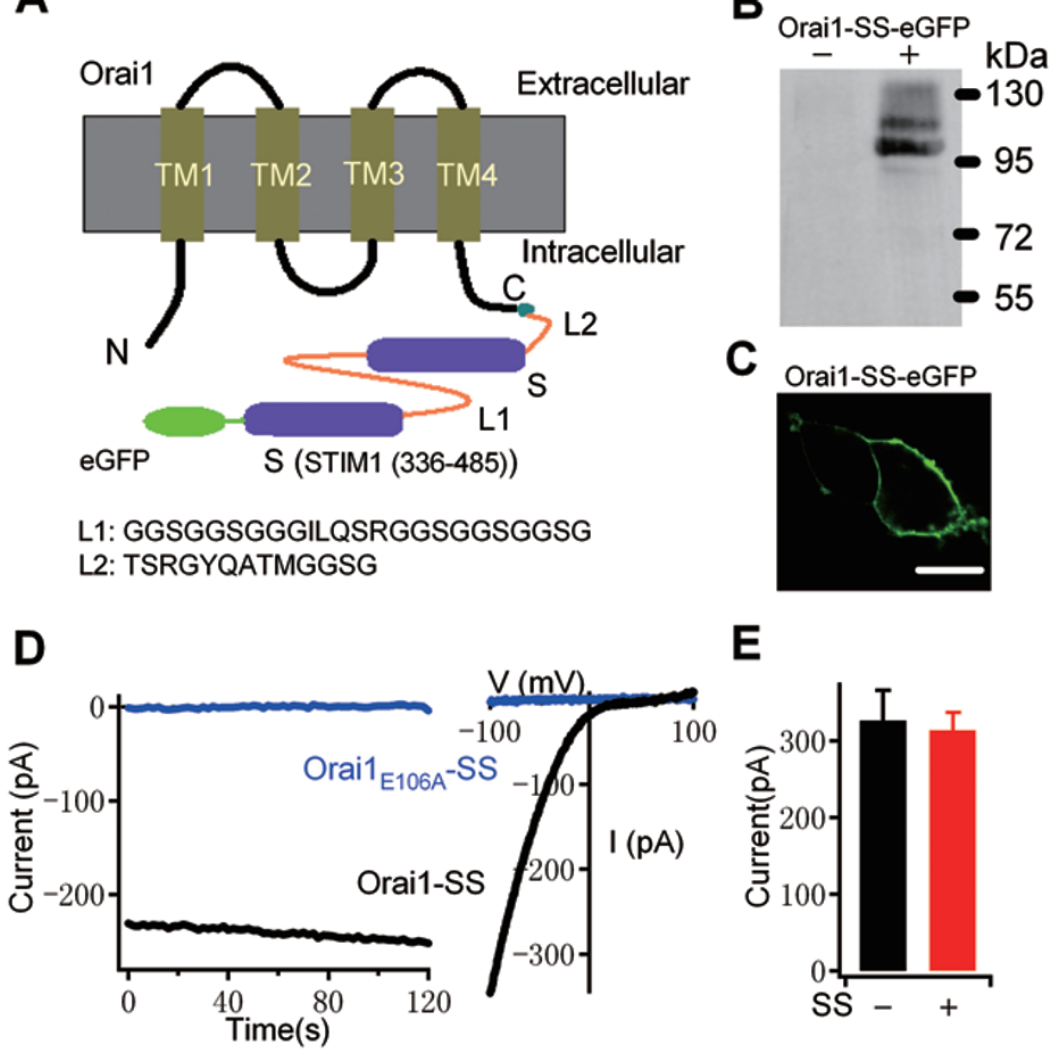

E

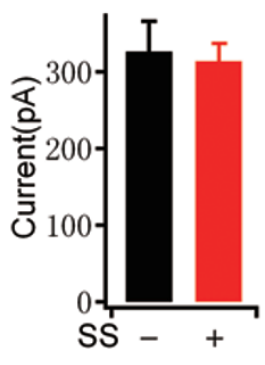

Figure 1 Orai1-SS generated constitutive, fully activated $I_{\text {CRAC. }}$ (A) Schematic diagram of Orai1-SS-eGFP. S, Stim1 (336-485). Tandem S domains (SS) were created by covalently fusing two $S$ domains with a 24-amino acid linker sequence (L1). The Orai1 subunit is attached intracellularly to SS using a 13-amino acid linker sequence (L2). The sequences of L1 and L2 are indicated at the bottom. (B) Western blots of non-tranfected cells and cells transfected with Orai1-SS-eGFP, visualized with GFP-specific antibody. (C) Location at the PM of Orai1-SS-eGFP in HEK293 cells. Images were captured at the middle plane using confocal microscopy. Scale bar, $10 \mu \mathrm{m}$. (D) Left: representative time courses of $\mathrm{I}_{\text {CRAC }}$ evaluated at $-80 \mathrm{mV}$ in $\mathrm{HEK} 293$ cells transfected with Orai1-SS-eGFP (black) or Orai $1_{\mathrm{E} 106 \mathrm{~A}}$-SS-eGFP (blue). Cells were bathed in external solution containing $6 \mathrm{mM} \mathrm{Ca}^{2+}$ and dialyzed with internal solution with free $\mathrm{Ca}^{2+}$ buffered to $145 \mathrm{nM}$ by $\mathrm{CaCl}_{2}$ and BAPTA. Currents were plotted after break-in. Right: characteristic leak-subtracted I-V relationships for $\mathrm{I}_{\text {CRAC }}$ recorded in the same cells from the left panel. Data were representative of more than five cells. (E) Additional SS did not contribute to $\mathrm{I}_{\mathrm{CRAC}}$ of Orai1-SS. Summary (mean \pm s.e.m.) of $\mathrm{I}_{\mathrm{CRAC}}$ amplitudes at $-100 \mathrm{mV}$ in cells expressing Orai1-SS-eGFP alone (black; $n=13$ ) or together with SS-mKO (red; $n=12)$. 
was recorded in cells expressing SS alone (Supplementary information, Figure S2), consistent with the low level of endogenous Orail in HEK293 cells. Then, we fused a single Orail subunit to the N-terminus of SS to generate an eGFP-tagged Orai1-SS (Orai1-SS-eGFP) with a stoichiometry of one Orail subunit and two S domains (Figure 1A). Western blotting of HEK293 cells transfected with Orail-SS-eGFP detected bands at $\sim 100$ $\mathrm{kDa}$, consistent with the predicted molecular mass of Orail-SS-eGFP and confirming that the intact full-length chimeric protein was properly expressed (Figure 1B). Orail-SS-eGFP was properly translocated to the PM as confirmed by confocal fluorescence imaging (Figure 1C). Using whole-cell patch clamp recording, large spontaneous currents with inwardly rectifying I-V curves were recorded in HEK293 cells expressing Orai1-SS-eGFP (Figure 1D). These currents shared many characteristics of native $\mathrm{I}_{\mathrm{CRAC}}$ current, such as an inwardly rectifying $\mathrm{I} /$ $\mathrm{V}$ relationship, dependence on extracellular $\mathrm{Ca}^{2+}$, permeability to $\mathrm{Na}^{+}$in divalent-free solution (DVF), sequential potentiation and inhibition by $100 \mu \mathrm{M} 2$-APB and blockade by $\mathrm{La}^{3+}$ (Supplementary information, Figure S3). No current was observed when wild-type (WT) Orail was substituted with a non-conducting Orai $1_{\mathrm{E} 106 \mathrm{~A}}$ mutant [9] (Figure 1D), confirming that the current was mediated by the Orail subunit in Orail-SS. Thus, this self-activated current indicates that the SS domain fused to Orail was not only correctly targeted, but also fully functional.

\section{CRAC channel formed by Orail-SS reaches the maximal activation level}

To test whether the CRAC channel formed by Orai1SS was maximally activated, currents in cells expressing Orail-SS-eGFP either alone or together with SS-monomeric Kushibara Orange (mKO) were compared. To control expression levels of Orail proteins, we selected cells with similar total GFP fluorescence (Supplementary information, Figure S4) for recording. We verified that the cell surface portion of Orail-containing chimeras was not altered by coexpression of SS domains (Supplementary information, Figure S4H). This fluorescencebased control of channel protein levels was employed throughout the study whenever comparison between different constructs was made (see Materials and Methods). We reasoned that if Orail-SS did not provide enough S domains to fully activate the CRAC channel, supplying additional $\mathrm{S}$ domains to the channel pore should augment the currents. However, the $\mathrm{I}_{\mathrm{CRAC}}$ produced by coexpression of Orail-SS-eGFP with SS-mKO showed a similar amplitude $(314 \pm 23 \mathrm{pA}, n=12)$ compared to that of Orail-SS-eGFP expressed alone $(327 \pm 40 \mathrm{pA}, n=13$; Figure $1 \mathrm{E})$, suggesting that CRAC channels formed by Orai1-SS reach the maximum. Coexpression of SSeGFP did not enhance the $\mathrm{I}_{\mathrm{CRAC}}$ produced by Orai1-SS fused with a smaller $3 \times$ Flag tag either (Supplementary information, Figure S5A). Increasing the linker length between the Orail and the SS domain to 44 amino acids (Orail- $\mathrm{L}_{44}-\mathrm{SS}$ ) generated similar currents in amplitude compared with the original Orai1-SS (Supplementary information, Figure S5B), indicating that neither the tag nor the linker perturb the channel activation. The attempt to link Orail with three tandem S domains was not successful as Orai1-SSS exhibited enhanced accumulation in intracellular compartments and reduced $\mathrm{I}_{\mathrm{CRAC}}$ amplitudes (Supplementary information, Figure S5C and S5D).

It is not known whether an Orai1-SS tetramer is crossactivated by $\mathrm{S}$ domains from a nearby channel rather than its own SS domains. To address this uncertainty, we coexpressed the non-conducting Orai $1_{\mathrm{E} 106 \mathrm{~A}} \mathrm{SS}$ eGFP with mKO-tagged four-tandem Orail (4×Orai1$\mathrm{mKO}$ ). Both constructs were trafficked to the PM. As the four-tandem Orai1 (4×Orai1) itself assembles to form a CRAC channel without interacting with other Orail subunits $[17,18], 4 \times$ Orail would be activated by $\mathrm{S}$ domains from Orai $1_{\mathrm{E} 106 \mathrm{~A}}-\mathrm{SS}$, if cross-interaction is possible. However, no $\mathrm{I}_{\mathrm{CRAC}}$ was recorded in cells coexpressing $4 \times$ Orail-mKO and Orai $1_{\mathrm{E} 106 \mathrm{~A}}-\mathrm{SS}-\mathrm{eGFP}$, as compared to large spontaneous $\mathrm{I}_{\mathrm{CRAC}}$ reconstituted by coexpression of $4 \times$ Orai1-mKO with SS-eGFP (Figure 2A). Therefore, these results exclude the possibility of a cross-activation of the Orail channel by $\mathrm{S}$ domains from neighboring Orail-SS protein, and indicate that Orail-SS functions through self-interaction inside an assembled channel.

\section{Both $S$ domains of Orail-SS contribute to the activation of Orail channel}

The linkers in Orail-SS (L1 and L2 in Figure 1A) might constrain the free movement of the $\mathrm{S}$ domains and hinder the interaction of either $\mathrm{S}$ domain with the Orail protein. To address this issue, we introduced the LQ347/348AA mutation in either S domain within Orai1SS-eGFP. The LQ347/348AA mutation has been shown to abolish the ability of STIM1 and SOAR to activate Orai1 [23]. Consistent with that report, overexpressing $\mathrm{S}_{\mathrm{LQ} / \mathrm{AA}}$ with Orail in HEK293 cells generated much reduced CRAC current as compared with cells transfected with WT S domain and Orail, despite the association of $\mathrm{S}_{\mathrm{LQ} / \mathrm{AA}}$ with Orail at the PM (Supplementary information, Figure S6). Similarly, Orai1- $\mathrm{S}_{\mathrm{LQ} / \mathrm{AA}} \mathrm{S}$ and Orai1-SS $\mathrm{LQ}_{\mathrm{LAA}}$ generated equally reduced constitutive $\mathrm{I}_{\mathrm{CRAC}}$ compared with Orai1-SS (Orai1-SS, $364 \pm 24$ pA, $n=10$; Orai1$\mathrm{S}_{\mathrm{LQ} / \mathrm{AA}} \mathrm{S}, 176 \pm 14 \mathrm{pA}, n=12$; Orai1-SS $\mathrm{LQ}_{\mathrm{LQ} / \mathrm{AA}}, 174 \pm 16$ $\mathrm{pA}, n=12$; Figure $2 \mathrm{~B}$ ). This suggests that there is no selectivity or priority in either S domain of Orail-SS for 

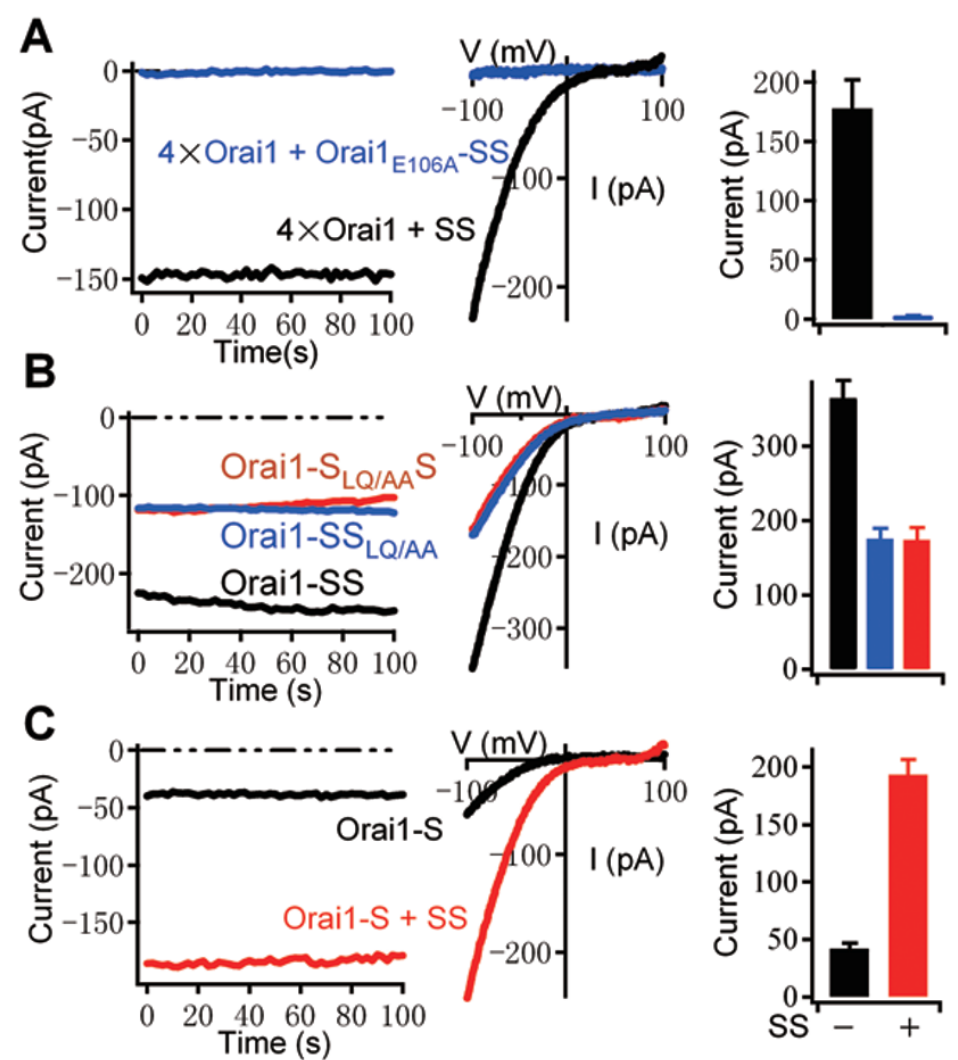

Figure 2 The CRAC channel formed by Orai1-SS is activated by the covalently associated S domains and both $S$ domains of Orai1-SS participate in activating the channel. (A) $S$ domains in Orai $1_{\mathrm{E} 106 \mathrm{~A}}-\mathrm{SS}$ could not activate channels formed by $4 \times \mathrm{Orai1}$. Left: time courses of $\mathrm{I}_{\mathrm{CRAC}}$ in cells expressing $4 \times$ Orai1-mKO together with either SS-eGFP (black) or Orai1 $1_{\mathrm{E} 106 \mathrm{~A}}-\mathrm{SS}-\mathrm{eGFP}$ (blue). Middle: representative I-V relationships form the same cells as in the left. Right: average current amplitudes at $-100 \mathrm{mV}$ of nine cells in each condition. (B) LQ/AA mutation in either S domain of Orai1-SS reduced the current amplitude equally. Representative time courses (left), I-V relationships (middle) and summary of current amplitudes (right) of cells transfected with

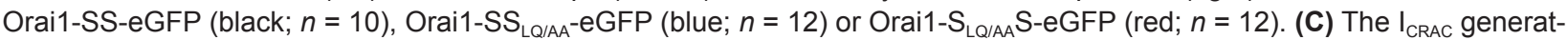
ed by Orai1-S was augmented by additional S domains. Representative time courses (left), I-Vs (middle) and average current amplitudes (right) of cells expressing Orai1-S-eGFP alone (black; $n=10$ ) or together with SS-mKO (red; $n=13$ ). Averaged data are presented as mean \pm s.e.m.

channel activation. Moreover, eGFP-tagged construct composed of one Orail subunit fused to one $\mathrm{S}$ domain (Orail-S-eGFP) generated reduced constitutive $\mathrm{I}_{\mathrm{CRAC}}(42$ $\pm 5 \mathrm{pA}, n=10)$ and this current was augmented by coexpression of SS-mKO $(193 \pm 14 \mathrm{pA}, n=13$; Figure $2 \mathrm{C})$, suggesting that the single $\mathrm{S}$ domain in Orail-S does not maximally open the associated Orail channel. These results indicate that both S domains of Orail-SS participate in the activation of the linked Orail channel.

$I_{C R A C}$ amplitude depends on the number of $S$ domains linked to Orail subunits

The reduced $\mathrm{I}_{\mathrm{CRAC}}$ generated by Orail-S as compared with Orai1-SS suggests that CRAC channel may not be maximally opened when presented with fewer than eight
$\mathrm{S}$ domains. To test this possibility, we fused SS to the two-tandem Orai1 $(2 \times$ Orail-SS) and to the four-tandem Orail (4×Orail-SS). As two-tandem Orail dimerizes to form a CRAC channel pore and four-tandem Orail forms a channel pore by itself [17], $2 \times$ Orail-SS and $4 \times$ Orai1SS would generate channels associated with four $\mathrm{S}$ and two $\mathrm{S}$ domains, respectively. Expression of full-length $2 \times$ Orail-SS-eGFP and $4 \times$ Orai1-SS-eGFP vectors was confirmed by western blotting (Figure $3 \mathrm{~A}$ ) and their location at the PM was determined by confocal imaging (Figure $3 \mathrm{~B})$. Similar to Orai1-S, cells expressing $2 \times$ Orai1SS-eGFP (Figure 3C) generated a small $\mathrm{I}_{\text {CRAC }}(45 \pm 3 \mathrm{pA}$, $n=12$ ). Coexpression of SS-mKO with $2 \times$ Orail-SSeGFP augmented the current amplitude $\sim 4$-fold $(195 \pm 23$ $\mathrm{pA}, n=12$; Figure 3C). Similar current amplitudes were 
A

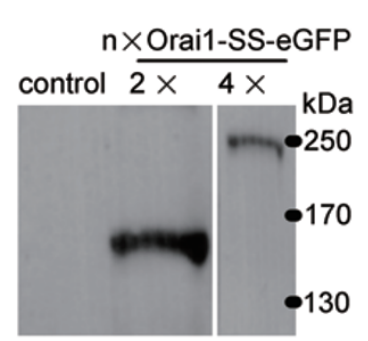

C

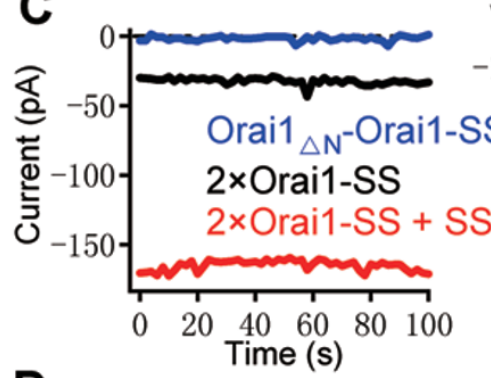

D

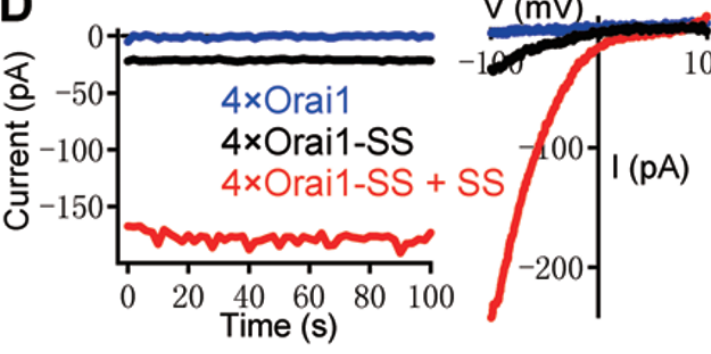

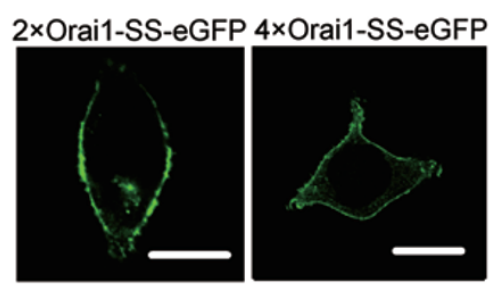

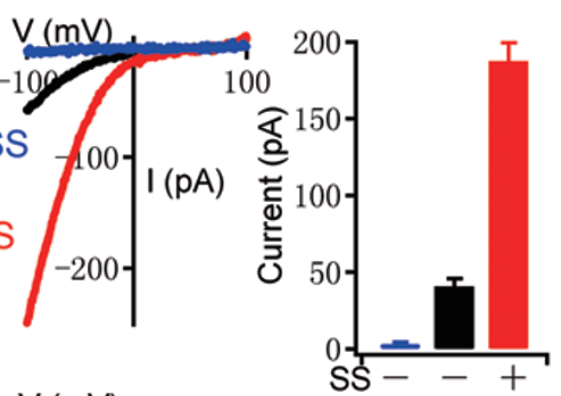

E

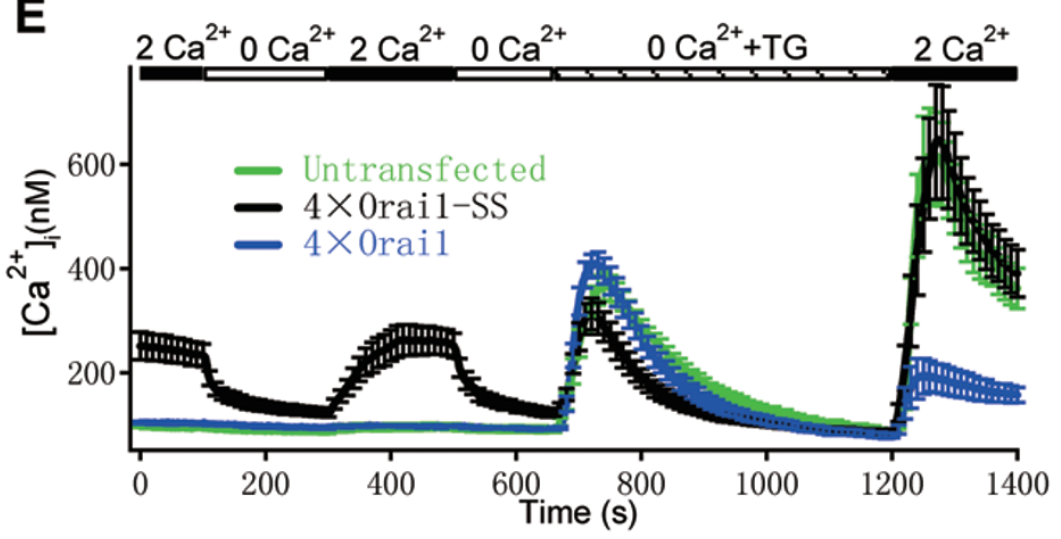

Figure 3 Partial activation of the CRAC channel by four and two $S$ domains. (A) Western blots of cells transfected with 2×Orai1-SS-eGFP or 4×Orai1-SS-eGFP, using GFP-specific antibody. Untransfected cells were used as control. (B) $2 \times$ Orai1SS-eGFP and $4 \times$ Orai1-SS-eGFP were dominantly located at the PM under confocal microscopy. Scale bars, $10 \mu \mathrm{m}$. (C) $2 \times$ Orai1-SS generated constitutive current and the current was augmented by additional SS. Left: representative time courses of $\mathrm{I}_{\mathrm{CRAC}}$ in cells expressing Orai $1_{\triangle \mathrm{N}}$-Orai1-SS-eGFP (blue), $2 \times$ Orai1-SS-eGFP (black) or $2 \times$ Orai1-SS-eGFP together with SS-mKO (red). Middle: characteristic I-Vs from the same cells in the left panel. Right: average current amplitudes (mean \pm s.e.m.) of cells transfected with Orai1 $1_{\Delta N}$-Orai1-SS-eGFP $(n=7)$, $2 \times$ Orai1-SS-eGFP $(n=9)$ or $2 \times$ Orai1-SS-eGFP together with SS-mKO $(n=10)$. (D) Time courses (left), I-Vs (middle) and averages (right; mean \pm s.e.m.) of $I_{\text {CRAC }}$ in cells expressing $4 \times$ Orai1-eGFP (blue; $n=11$ ), $4 \times$ Orai1-SS-eGFP (black; $n=11$ ) or $4 \times$ Orai1-SS-eGFP together with SS-mKO (red; $n=13)$. (E) $4 \times$ Orai1-SS activated $\mathrm{Ca}^{2+}$ influx without depleting intracellular $\mathrm{Ca}^{2+}$ stores. Mean \pm s.e.m. of $\mathrm{Ca}^{2+}$ measurements in untransfected HEK293 cells (green; $n=15$ ) and cells expressing $4 \times$ Orai1-eGFP (blue; $n=15$ ), $4 \times$ Orai1-SS-eGFP (black; $n=19$ ) are shown. Solution exchange is indicated by the bars. $1 \mu \mathrm{M} \mathrm{TG}$ in $\mathrm{Ca}^{2+}$-free solution was used to deplete $\mathrm{Ca}^{2+}$ stores. 
observed when the linker between the two-tandem Orail and SS was lengthened to 44 amino acids (Supplementary information, Figure S7A and S7B). It remains possible that four $2 \times$ Orail-SS interact with each other and each contributes one Orail subunit to form a channel pore with eight interacting S domains. Therefore, we generated a construct with the $\mathrm{N}$-terminus (residues 1-90) of the first Orail subunit truncated (Orai $1_{\Delta N^{-}}$Orail-SSeGFP), which was unable to conduct current as shown previously. Arguing against that possibility, overexpressing Orai1 ${ }_{\Delta N}$-Orai1-SS in HEK293 cells failed to reconstitute any currents (Figure 3C), although it was efficiently trafficked to the PM (Supplementary information, Figure S7G). These results suggest that four $\mathrm{S}$ domains do open the CRAC channel and more $\mathrm{S}$ domains are required to maximize $\mathrm{I}_{\mathrm{CRAC}}$. Similarly, patch clamp experiments revealed small spontaneous $\mathrm{I}_{\mathrm{CRAC}}$ in cells expressing $4 \times$ Orail-SS-eGFP (Figure $3 \mathrm{D}$ ) or $4 \times{\text { Orail } 1-\mathrm{L}_{44}-\mathrm{SS}}$ (Supplementary information, Figure S7D and S7E). The current generated by $4 \times$ Orail-SS-eGFP was enhanced by about 9 -fold ( $179 \pm 15 \mathrm{pA}, n=13)$ by coexpression of SS-mKO (Figure 3D). No $\mathrm{I}_{\text {CRAC }}$ was observed in cells expressing $4 \times$ Orail-eGFP alone (Figure 3D), indicating that the small current did not result from spontaneous activation of the concatenated Orail tetramer by endogenous STIM1. We also measured free cytoplasmic $\mathrm{Ca}^{2+}$ concentration $\left(\left[\mathrm{Ca}^{2+}\right]_{i}\right)$ to further examine the store-operated $\mathrm{Ca}^{2+}$ entry (SOCE) in cells expressing these vectors. Compared with untransfected cells and cells transfected with $4 \times$ Orail-eGFP, cells expressing $4 \times$ Orail-SS-eGFP showed higher basal $\left[\mathrm{Ca}^{2+}\right]_{i}$ and spontaneous $\mathrm{Ca}^{2+}$ influx without depleting intracellular stores (Figure 3E), in full agreement with the electrophysiological recordings and our previous finding [17]. In addition, after thapsigargin (TG)-induced depletion of $\mathrm{Ca}^{2+}$ from the ER, cells transfected with $4 \times$ Orai1-SS-eGFP showed slightly larger $\mathrm{Ca}^{2+}$ influx. In contrast, SOCE in cells transfected with $4 \times$ Orai1-eGFP was largely suppressed (Figure $3 \mathrm{E}$ ), similar to previous reports that overexpression of WT Orail inhibited endogenous $\mathrm{I}_{\mathrm{CRAC}}[14,25,26]$.

Graded activation of CRAC channel by activating different numbers of Orail subunits

To test directly whether the multiple levels of CRAC current resulted from different numbers of Orail subunit being activated, we sought to find a mutant that selectively perturb the activation of individual subunit in the channel complex. The mutation of Orail at position 273 (L273S) has been reported to prevent the interaction of the cytosolic C-terminus of Orail with STIM1 and hence the activation by STIM1 [27]. Similarly, coexpression of SS or STIM1 with Orai $1_{\text {L273S }}$ failed to generate $\mathrm{I}_{\mathrm{CRAC}}$ current in our hands (Supplementary information, Figure S8A and S8B). To our surprise, Orai $1_{\mathrm{L} 273 \mathrm{~S}}$-SS exhibited large constitutive $\mathrm{I}_{\mathrm{CRAC}}$ (Supplementary information, Figure $\mathrm{S} 8 \mathrm{C}$ ), while the $\mathrm{I}_{\mathrm{CRAC}}$ amplitudes decreased when the linker between Orai1 $1_{\mathrm{L} 273 \mathrm{~S}}$ and SS is lengthened to 44 amino acids (Supplementary information, Figure S8D). These results imply that the short linker might force the SS domain to interact with Orai $1_{\text {L273S }}$ despite a reduced affinity. We speculated that a mutation of L273 to a polar, charged amino acid would be stronger than the L273S mutation and might abolish the activation of Orail by the linked S domains. Indeed, unlike Orai1 $1_{\mathrm{L} 273 \mathrm{~S}}-\mathrm{SS}$, tethered SS domains (Orai $1_{\text {L273D }}-\mathrm{SS}$ ) were not able to activate Orai $1_{\mathrm{L} 273 \mathrm{D}}$, though Orai $1_{\mathrm{L} 273 \mathrm{D}}$-SS was efficiently trafficked to the PM (Figure 4A and 4C). In addition, soluble SS could not be recruited to the PM by Orai $1_{\mathrm{L} 273 \mathrm{D}}$, and expression of Orai $1_{\text {L273D }}$ together with either SS or STIM1 did not generate observable $\mathrm{I}_{\mathrm{CRAC}}$ (Figure 4B, 4D and $4 \mathrm{E})$. These results suggest that the L273D replacement in Orai1, like its forerunner L273S, disrupts the proper interaction between Orai1 and STIM1, and thus abolishes the channel activation.

Therefore, we introduced the L273D mutation in the $4 \times$ Orail-SS-eGFP to generate a series of constructs containing one (Orai $1_{\mathrm{L} 273 \mathrm{D}^{-}}-3 \times$ Orai1-SS $)$, two $\left(2 \times\right.$ Orai $_{\mathrm{L}_{273 \mathrm{D}^{-}}}$ $2 \times$ Orai1-SS), three $\left(3 \times\right.$ Orai $_{\mathrm{L} 273 \mathrm{D}}-$ Orai1-SS $)$ and four mutated (mut) subunits $\left(4 \times\right.$ Orai $\left._{\text {L273D }}-S S\right)$. Channels composed of different combination of mut and WT Orai1 subunits could thus be produced. Patch clamp recording of cells expressing each of these constructs alone or together with SS-mKO showed the following (Figure 5A): (1) Overexpression of $4 \times \mathrm{Orai}_{\mathrm{L} 273 \mathrm{D}}-\mathrm{SS}$ alone or with SS in HEK293 cells failed to reconstitute $\mathrm{I}_{\text {CRAC }}$, consistent with the inability of Orai1 $1_{\mathrm{L} 273 \mathrm{D}}$ to interact with SS domains. (2) Expression of $3 \times$ Orai $_{\mathrm{L} 273 \mathrm{D}}-$ Orai1-SS, $2 \times$ Orai $_{\mathrm{L}_{273 \mathrm{D}}}-2 \times$ Orai1-SS and Orai $1_{\mathrm{L} 273 \mathrm{D}}-3 \times$ Orai1-SS alone generated currents with similar amplitudes to those generated by expression of $4 \times$ Orail-SS, indicating that the current was generated by a single Orail bound with SS in the CRAC channel. (3) Coexpression of SS did not augment the $\mathrm{I}_{\mathrm{CRAC}}$ of $3 \times$ Orai $_{\mathrm{L} 273 \mathrm{D}}$-Orai1-SS but led to graded increase in the $\mathrm{I}_{\mathrm{CRAC}}$ of $2 \times$ Orai $_{\mathrm{L} 273 \mathrm{D}}-2 \times$ Orail-SS,

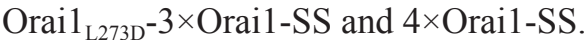

To examine whether full-length STIM1 also opened the CRAC channel upon $\mathrm{Ca}^{2+}$ store depletion in a graded fashion, we utilized a series of four-tandem heterozygous mut/WT Orail constructs that contained one (Orai $1_{\text {L273D }}-3 \times$ Orai1), two $\left(2 \times\right.$ Orai $_{L_{2273 D}}-2 \times$ Orai1 $)$, three $\left(3 \times\right.$ Orai $_{\text {L273D }}-$ Orai1) and four Orai1 $1_{\text {L273D }}$ subunits $\left(4 \times\right.$ Orai $\left._{\text {L273D }}\right)$. Each of these constructs or $4 \times$ Orai1-eGFP was coexpressed with mOrange-tagged STIM1 (STIM1mOrange) in HEK293 cells. Except for the $4 \times$ Orai $_{\text {L273D, }}$ 

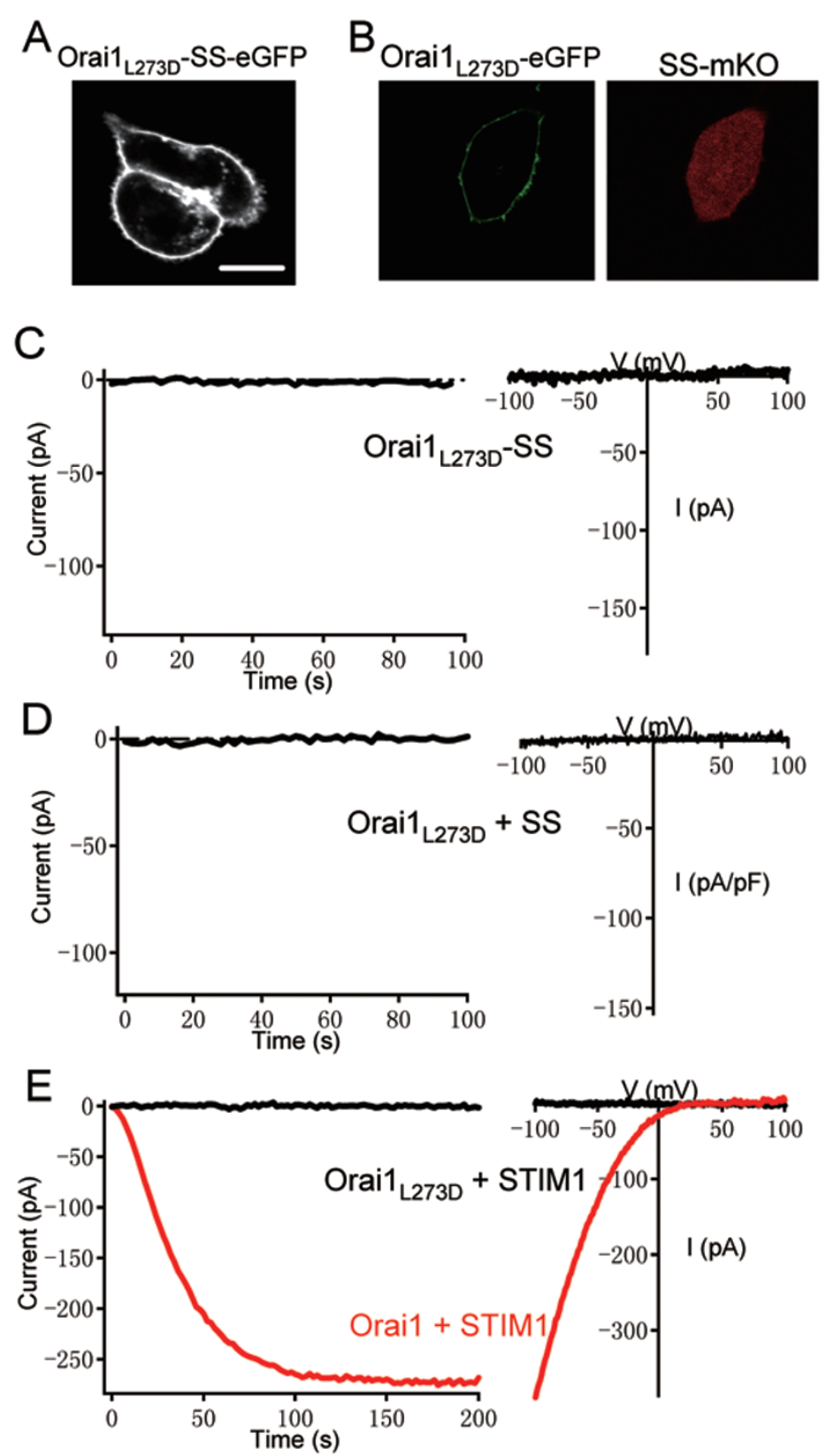

Figure 4 L273D mutation of Orai1 abolishes the $\mathrm{I}_{\mathrm{CRAC}}$ of Orai1-SS. (A) Orai1 $1_{\mathrm{L} 273 \mathrm{D}}-\mathrm{SS}$-eGFP was efficiently translocated to the plasma membrane as shown by confocal imaging. (B) Orai1 ${ }_{\mathrm{L} 273 \mathrm{D}}$-eGFP was efficiently translocated to the PM (left) but could not recruit coexpressed SS-mKO (right). Images were captured at the middle plane using confocal microscopy. (C) Orai $1_{\mathrm{L}_{273 \mathrm{D}^{-}}}$ SS did not produce self-activated $\mathrm{I}_{\text {CRAC }}$. Representative time courses (left) and I-V relationships (right) of cells transfected with Orai $1_{\mathrm{L} 273 \mathrm{D}}-\mathrm{SS}-\mathrm{eGFP}$. (D) No $\mathrm{I}_{\mathrm{CRAC}}$ was observable in cells cotransfected with SS-mKO and Orai $1_{\mathrm{L} 273 \mathrm{D}}-\mathrm{eGFP}$. (E) Orai $1_{\mathrm{L} 273 \mathrm{D}}$ could not be activated by STIM1 after store depletion. Cells were cotransfected with STIM1-mOrange + Orai1-eGFP (red) or STIM1-mOrange + Orai1 ${ }_{\text {L273D }}$-eGFP (black). Data are representatives of more than three cells in each condition.

all the other constructs reconstituted $\mathrm{I}_{\mathrm{CRAC}}$ in patch clamp experiments with a pipette solution containing $10 \mu \mathrm{M}$ inositol 1,4,5-trisphosphate $\left(\operatorname{Ins}(1,4,5) \mathrm{P}_{3}\right)$ to deplete $\mathrm{Ca}^{2+}$ stores (Figure 5B and 5C). The $\mathrm{I}_{\text {CRAC }}$ amplitudes increased as the number of WT subunits in the vector in- creased (Figure 5D): $21 \pm 4.0 \mathrm{pA}$ for $3 \times$ Orai $_{\mathrm{L} 273 \mathrm{D}}-$ Orai1, $61 \pm 12 \mathrm{pA}$ for $2 \times$ Orai $_{\mathrm{L} 273 \mathrm{D}}-2 \times$ Orai $1,157 \pm 25 \mathrm{pA}$ for Orai $1_{\mathrm{L} 273 \mathrm{D}}-3 \times$ Orail and $290 \pm 43 \mathrm{pA}$ for $4 \times$ Orai1. Thus, graded augmentation of CRAC channel is not limited to SS domains, but also occurs with full-length STIM1 un- 


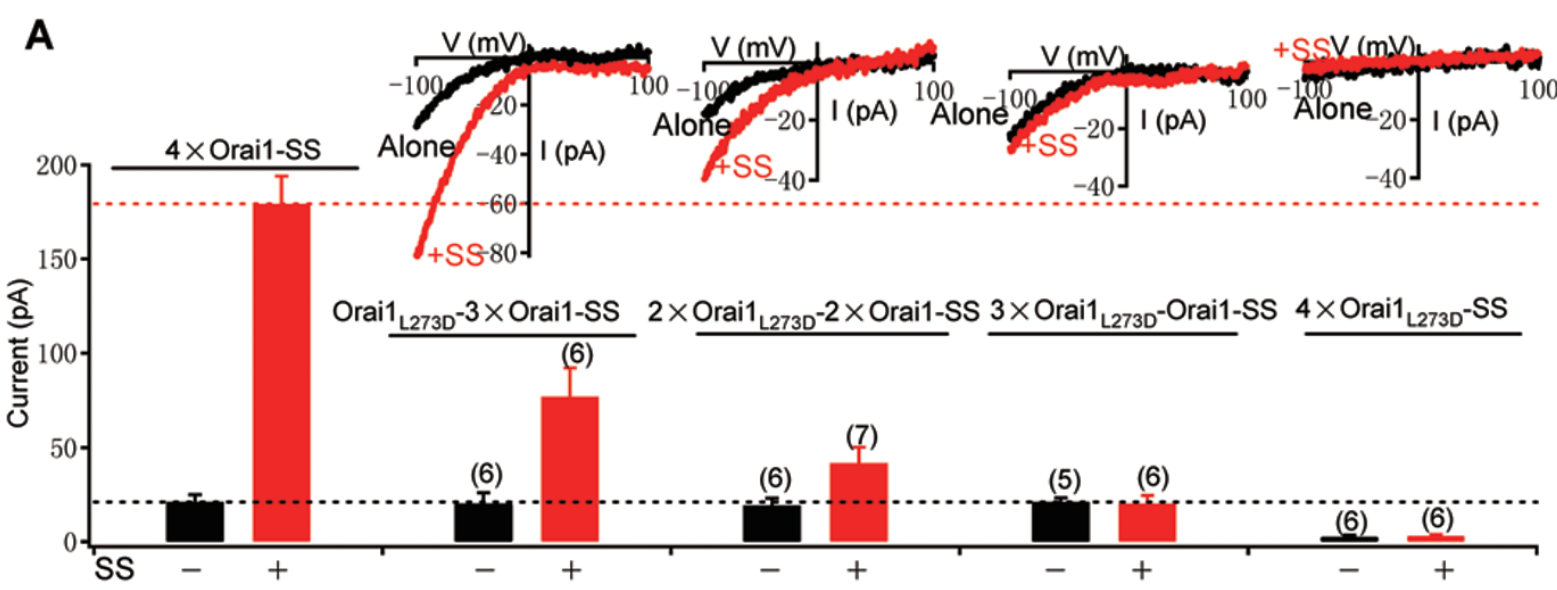

B

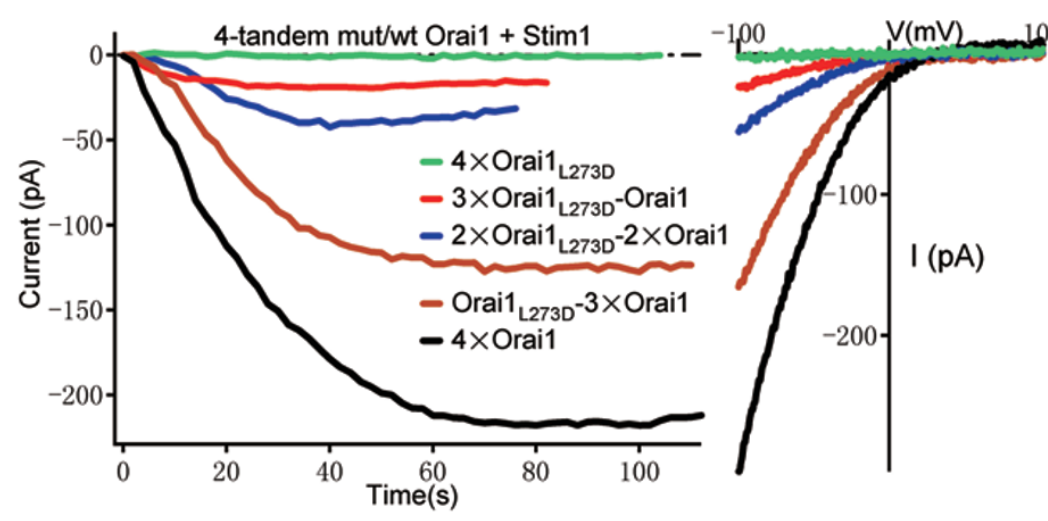

D

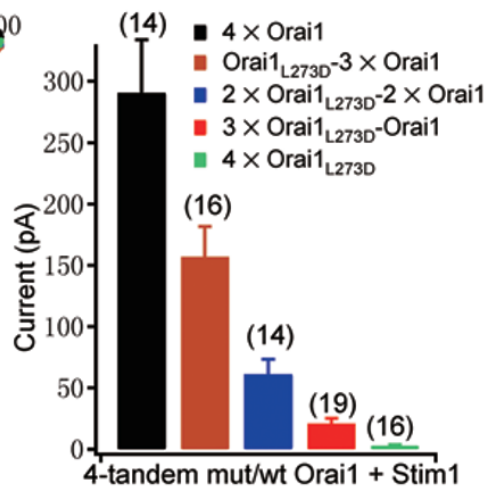

Figure 5 Two $S$ domains open the Orai1 channel through specifically interacting with one Orai1 subunit, and coexpressed $S$ domains or STIM1 increases $I_{\text {CRAC }}$ gradually with the increment of WT Orai1 subunits in the channel. (A) Representative I-V relationships (upper) and averages (lower; mean \pm s.e.m.) of cells expressing each of the four indicated eGFP-tagged constructs alone (black) or together with SS-mKO (red). Numbers of cells in each category are labeled in brackets above the columns. For comparison, $I_{\text {CRAC }}$ averages of $4 \times$ Orai1-SS (black) or $4 \times$ Orai1-SS $+S S$ (red) reproduced from Figure 3D. (B) Developments of $\mathrm{I}_{\mathrm{CRAC}}$ in cells cotransfected with Stim1-mOrange and each of the eGFP-tagged four-tandem Orai1 constructs containing 0-4 Orai $1_{\mathrm{L} 273 \mathrm{D}}$ subunits. $10 \mu \mathrm{M} \operatorname{Ins}(1,4,5) \mathrm{P}_{3}$ in pipette solution was used to deplete $\mathrm{Ca}^{2+}$ store. (C) I-Vs at maximal activation for each category as marked in (B). (D) Summary (mean \pm s.e.m.) of peak current amplitudes for each category as indicated. Numbers of cells are labeled in the brackets.

der physiological stimulation.

\section{Discussion}

In the current study, we took an approach to interrogate the stoichiometry of CRAC channel by linking different numbers of Orail and functional $\mathrm{S}$ domains of STIM1 and reconstituting CRAC-like channels in HEK293 cells. Our results demonstrate that channels composed of four Orai1 and tethered eight $\mathrm{S}$ domains gave rise to the maximum current, which cannot be further increased by providing more soluble SS domains. Although tethering has the advantage of providing high local concentration of proteins, it may limit the effectiveness of tethered proteins by exerting geometric constraints. In our case, we believe that tethered SS domains were, at least, of the same potency in binding to and activating Orail channels as the soluble SS domains or fulllength STIM1 for the following reasons: first, Orai1-SS generated similar currents in amplitude as Orail coexpressed with SS or full-length STIM1 (Supplementary information, Figure S9A). Second, coexpression of SS did not augment the current of Orai1-SS $\mathrm{LQ}_{\mathrm{LQAA}}$ (Supplementary information, Figure S9B). Finally, channels formed by the Orai1 $1_{\text {L273S }}$ mutants could neither be activated by coexpressed SS (Supplementary information, Figure S8A), 
nor by full-length STIM1 after store depletion (Supplementary information, Figure S8B) [27]. In contrast, tethering SS domain to Orai $1_{\mathrm{L} 273 \mathrm{~S}}$ produced huge CRAC currents, which were reduced when the linker between Orai1 and SS domain was increased to $44 \mathrm{AA}$ (Orai $1_{\mathrm{L}_{273 \mathrm{~S}^{-}}}$ $\mathrm{L}_{44}$-SS; Supplementary information, Figure S8C). This result suggests that tethered SS domains are indeed more efficient in activating the Orail channel than the soluble $\mathrm{S}$ domains by providing a high local concentration of $\mathrm{S}$ domain in the close proximity to Orail channel.

To further demonstrate whether all tethered eight $\mathrm{S}$ domains or only a portion of them contribute to the activation of tandem Orail chimeras, we sought to evaluate the relative contribution of each tethered SS domain. We found that the L273D mutation of Orail abolished the interaction between Orail and SS domains and hence the generation of $\mathrm{I}_{\mathrm{CRAC}}$ (Figure 4). We then mixed different numbers of non-interacting Orai $1_{\mathrm{L} 273 \mathrm{D}}$ mutants and WT Orail subunits, and evaluated the contribution of SS binding to each Orail monomer in generating $\mathrm{I}_{\mathrm{CRAC}}$. Our data support that two $\mathrm{S}$ domains specifically interact with one Orail subunit and $\mathrm{I}_{\mathrm{CRAC}}$ increases gradually with increment of WT Orail subunits upon binding of S domains. Based on above results, we propose that two STIM1 molecules can open the CRAC channel through binding to one Orail subunit; the opening activity of the channel increases with more Orail subunits bound with STIM1 and channel reaches maximal activation when all four subunits are bound with eight STIM1 molecules. This is consistent with the results that STIM1 dimerizes through its cytosolic domain at rest [21], and dimeric cytosolic domains of STIM1 can efficiently activate the Orail channel $[28,29]$. However, we cannot, of course, completely exclude the possibility that the linkers in our constructs render the $\mathrm{S}$ domains to function differently from WT STIM1 and therefore the CRAC channel may have an alternative Orail-STIM1 stoichiometry.

The graded activation of CRAC channel by binding of different numbers of STIM1 helps to explain a long standing phenomenon in the field that overexpression of Orail inhibited endogenous SOCE in HEK293 cells (Figure 3E) $[14,25,26]$ and also $\mathrm{I}_{\mathrm{CRAC}}$ in RBL and Jurkat cells $[26,30]$. Our results would predict such an inhibitory effect as the availability of endogenous STIM1 for each Orail subunit is reduced upon overexpression of Orai1. Our data (see Figure 3 and Figure 5) indicate that the reduction in the number of available STIM1 for Orail should render a nonlinear, much greater decrease in the $\mathrm{I}_{\mathrm{CRAC}}$ amplitudes.

The graded activation has been reported for other tetrameric channels like the potassium channel [31], the AMPA receptor $[32,33]$ and the cyclic nucleotide gated
(CNG) channel [34]. In contrast to the fact that at least two ligand-bound subunits are required to significantly open the AMPA receptor and the CNG channel, as determined by single channel recording [32, 34], we find that the CRAC channel is partially activated at the macroscopic whole-cell level when only one Orail subunit can interact with STIM1. It is unknown which factor the opening probability, conductance or both of them - is changed during this graded activation process due to the undetectable single channel conductance. Nevertheless, such graded activation would enable the CRAC channel to generate a wide range of $\mathrm{I}_{\mathrm{CRAC}}$ corresponding to different numbers of STIM1 molecules available, as compared to an "all-or-none" activation mode (the channel opens only when all four subunits are interacting with STIM1). For example, when the ER $\mathrm{Ca}^{2+}$ store is partially depleted, which may be the case for most physiological stimulations, only a small amount of STIM1 dissociate with $\mathrm{Ca}^{2+}$ and become free to interact with Orai1; the graded activation mechanism we revealed here can ensure the opening of CRAC channels and hence refilling of $\mathrm{Ca}^{2+}$ store without concerted binding of all four Orail subunits to STIM1. The generation of multiple levels of $\mathrm{I}_{\mathrm{CRAC}}$ might also contribute to variable $\left[\mathrm{Ca}^{2+}\right]_{i}$ levels and oscillation patterns required for differential gene expressions in immune cells $[35,36]$.

\section{Materials and Methods}

\section{Cell culture and transfection}

HEK293 cells (ATCC) were cultured in Dulbecco's modified Eagle's medium (DMEM) with 10\% heat-inactivated fetal bovine serum, $50 \mathrm{U} / \mathrm{ml}$ penicillin and $50 \mathrm{mg} / \mathrm{ml}$ streptomycin. Cells were transfected with $1.6 \mu \mathrm{g}$ DNA using Lipofectamine 2000 (Invitrogen) reagent following the manufacturer's instructions. When the plasmids could generate constitutive $\mathrm{I}_{\mathrm{CRAC}}, 10 \mu \mathrm{M} \mathrm{LaCl}_{3}$ was added to the culture medium after transfection to avoid the toxicity of spontaneous $\mathrm{Ca}^{2+}$ influx. For coexpression experiments, SSmKO or SS-eGFP was cotransfected with Orail-derived constructs containing single Orail at a mass ratio of $1: 1$, that containing twotandem Orail at a ratio of $1: 2$ and that containing four-tandem Orail at a ratio of 1:5. Stim1-mOrange and four-tandem mut/WT Orail constructs were cotransfected at a ratio of 1:5. Expression of both constructs in each cell was confirmed by the emission fluorescence of eGFP and that of mKO or mOrange. For each set of experiments, the GFP fluorescence of Orail-derived constructs was measured to confirm that the differences in current amplitudes were not due to differences in expression levels of Orail channels (Supplementary information, Figure S4).

\section{Plasmids construction}

For the construction of SS-eGFP, the S domain (AAs 336-485 of STIM1) was amplified by PCR using the primers: 5'-GAG CTC GAG GGG GTA CCA AGC CAC CAT GGG TGG ATC CGG CGA ATC TCA CAG CTC ATG G-3' and 5'-CCG GAT CCC GAA 
TTC CAC CTC CGC TAC CTC CAG AGC CGC CCA CAA TCT CCT CAT CCA TC-3'. The fragment was then digested with XhoI $\left(5^{\prime}\right)$ and EcoRI (3') and inserted into a pEGFP-N1 (Clontech) vector. Then, the other S fragment was PCR amplified using another forward primer, 5'-GAG CTC GAG GGG TGG ATC CGG TGG GTC CGG CGG ATC CGG CGA ATC TCA CAG CTC ATG G-3' and the same reverse primer as the first $\mathrm{S}$ fragment. This fragment was then inserted downstream of the former-constructed $\mathrm{S}$ using SalI (5') and BamHI (3'). A linker sequence of 24 residues was thus generated between the tandem $\mathrm{S}$ domains. The mKO-tagged SS was produced by substituting the eGFP sequence with the $\mathrm{mKO}$ sequence in the $\mathrm{pEGFP}-\mathrm{N} 1$ vector.

Construction of Orai1, $2 \times$ Orail and $4 \times$ Orail tagged with eGFP or $\mathrm{mKO}$ was described previously [17], where an 18-residue linker sequence (MLHPGLSPGLLPLHPASI) was inserted immediately upstream of the N-terminus of each Orail sequence. Construction of mOrange-tagged STIM1 was described elsewhere [25].

For the constructions of eGFP-tagged Orail-S, Orai1-SS, $2 \times$ Orail-SS and $4 \times$ Orail-SS, NheI ( $\left.5^{\prime}\right)$ and SalI ( $\left.3^{\prime}\right)$ flanked Orai1, $2 \times$ Orail and $4 \times$ Orail were cut out from the plasmids containing Orai1-eGFP, $2 \times$ Orail-eGFP and $4 \times$ Orail-eGFP. These DNA fragments were ligated into the S-pEGFP-N1 or SS-pEGFP-N1 digested by NheI $\left(5^{\prime}\right)$ and XhoI $\left(3^{\prime}\right)$.

All point mutations were generated on single $\mathrm{S}$ or single Orail sequence using the standard site-directed mutation protocol and confirmed by sequencing. These mutants were then used to produce constructs containing proper arrangements and numbers of mutant subunits (See Supplementary information, Data S1 for the constructions of other plasmids used in this study).

\section{Western blotting}

$48 \mathrm{~h}$ after transfection, HEK293 cells were washed with PBS and lysed in SDS loading buffer. For Orail-SS-eGFP in Figure $1 \mathrm{~B}$, samples were denatured at $100{ }^{\circ} \mathrm{C}$ for $3 \mathrm{~min}$. For $2 \times$ Orail-SSeGFP and $4 \times$ Orail-SS-eGFP in Figure $3 \mathrm{~A}$, samples were loaded without denaturation at $100{ }^{\circ} \mathrm{C}$. Blots were probed with anti-GFP (Santa Cruz Biotechnology, Santa Cruz, CA) antibody according to the standard procedures.

\section{Confocal microscopy}

Transfected HEK293 cells were imaged under a confocal laser scanning microscope FV500 (Olympus Optical Co., Tokyo, Japan) in $\mathrm{Ca}^{2+}$-free Ringer's solution containing $155 \mathrm{mM} \mathrm{NaCl}, 4.5 \mathrm{mM}$ $\mathrm{KCl}, 2 \mathrm{mM} \mathrm{MgCl}$, $1 \mathrm{mM}$ EGTA, $10 \mathrm{mM}$ D-glucose and $5 \mathrm{mM}$ HEPES ( $\mathrm{pH}$ 7.4). All experiments were performed at room temperature.

\section{Electrophysiology}

Patch clamp experiments were performed at room temperature using standard whole-cell recording configuration as previously described [25]. Cells were plated on poly-L-lysine-coated coverslips 12-24 h before experiments. The extracellular solution contained $140 \mathrm{mM}$ cesium glutamate, $6 \mathrm{mM} \mathrm{CaCl}_{2}, 2 \mathrm{mM} \mathrm{MgCl}_{2}, 10$ $\mathrm{mM}$ tetraethylammonium chloride and $10 \mathrm{mM}$ Hepes $(\mathrm{pH} 7.4$, adjusted with $\mathrm{CsOH}$ ). For recording of constitutive $\mathrm{I}_{\mathrm{CRAC}}$, the pipette was filled with solution containing $140 \mathrm{mM}$ cesium glutamate, 8 $\mathrm{mM} \mathrm{MgCl}, 10 \mathrm{mM}$ BAPTA, $3.5 \mathrm{mM} \mathrm{CaCl}_{2}$ and $10 \mathrm{mM}$ Hepes (pH 7.2, adjusted with $\mathrm{CsOH}$ ). The free $\left[\mathrm{Ca}^{2+}\right]$ of this solution was calculated to be $145 \mathrm{nM}$ using MaxChelator. The pipette solution was added with $10 \mu \mathrm{M} \operatorname{Ins}(1,4,5) \mathrm{P}_{3}$ in some experiments (Figure $4 \mathrm{E}$ and Figure $5 \mathrm{~B}$ ) to deplete the $\mathrm{Ca}^{2+}$ stores. Before patch clamp recording, the GFP epifluorescence of each cell was excited by a xenon light source at $488 \mathrm{~nm}$ and measured at $510 \pm 5 \mathrm{~nm}$ with a photodiode. After establishment of whole-cell configuration, voltage stimuli consisting of a $10-\mathrm{ms}$ step to $-100 \mathrm{mV}$ followed by a 50 -ms voltage ramp from -100 to $+100 \mathrm{mV}$ were delivered from the holding potential of $0 \mathrm{mV}$ every $2 \mathrm{~s}$. Currents were digitized at a rate of $20 \mathrm{kHz}$ and filtered offline at $2 \mathrm{kHz}$. All current traces were leak-subtracted. I-V currents were collected by delivering voltage ramps after seal formation and before break-in, and that with current magnitude at $+50 \mathrm{mV}$ equal to the sustained outward current after break-in was assigned as leak current. Capacitive currents were determined and corrected before each voltage ramp. The current amplitude at $-80 \mathrm{mV}$ of individual ramp was extracted to monitor the development of $\mathrm{I}_{\mathrm{CRAC}}$ and current amplitudes at $-100 \mathrm{mV}$ were used for statistical analysis. Data were analyzed by IGOR Pro 5.01 (Wavemetrics). Averaged results were presented as the mean values \pm s.e.m. with the numbers of experiments indicated.

\section{Single cell $\left[\mathrm{Ca}^{2+}\right]_{i}$ measurement}

HEK293 cells were pre-incubated with $4 \mu \mathrm{M}$ fura-2/AM (Invitrogen) at $37{ }^{\circ} \mathrm{C}$ for $20 \mathrm{~min}$ in DMEM. The standard extracellular Ringer's solution contained $145 \mathrm{mM} \mathrm{NaCl}, 4.5 \mathrm{mM} \mathrm{KCl}, 2 \mathrm{mM}$ $\mathrm{CaCl}_{2}, 1 \mathrm{mM} \mathrm{MgCl} 2,10 \mathrm{mM}$ D-glucose and $5 \mathrm{mM}$ Hepes ( $\mathrm{pH} 7.4$, adjusted with $\mathrm{NaOH}$ ). The $\mathrm{CaCl}_{2}$ was replaced by 1 mM EGTA and $2 \mathrm{mM} \mathrm{MgCl}_{2}$ in $\mathrm{Ca}^{2+}$-free solution. Fluorescence of fura- 2 was excited by a dual wavelength excitation $(340 / 380 \mathrm{~nm})$ photometry system on an inverted microscope (IX71, Olympus, Tokyo, Japan) with a polychromatic xenon light source (TILL photonics, Gräfeling, Germany). The emission from a single cell was collected at $510 \pm 5 \mathrm{~nm}$ with a photodiode controlled by the TILL photometry system and X-Chart extension of Pulse software (HEKA, Lambrecht, Germany). $\left[\mathrm{Ca}^{2+}\right]_{i}$ was calculated using fluorescence ratio $\mathrm{R}(340 / 380)$ according to equation $\left[\mathrm{Ca}^{2+}\right]_{i}=K_{\mathrm{eff}}\left(R-R_{\min }\right) /\left(R_{\max }-R\right)$ as previously described [25].

\section{Conflict of interest}

The authors declare no competing financial interests. Requests for materials should be addressed to TX (xutao@ibp.ac.cn).

\section{Acknowledgments}

We thank Professor Roger Y Tsien (University of California at San Diego, La Jolla) for providing the mOrange; Drs Bertil Hille, Erwin Neher, Michael Cahalan and Joy Fleming for careful reading and critical comments on the manuscript; Li Zheng and Junbing Wu for advice on western blotting; Drs Xiaolan Xu, Yong $\mathrm{Yu}$ and Lijun Chen for discussion during the project. This work was supported by grants from the National Science Foundation of China (30870564, 30630020), the Major State Basic Research Program of China (2006CB911001, 2010CB833701) and the CAS Project (YZ200838).

\section{References}

1 Lewis RS. Calcium signaling mechanisms in T lymphocytes. 
Annu Rev Immunol 2001; 19:497-521.

2 Parekh AB, Putney JW Jr. Store-operated calcium channels. Physiol Rev 2005; 85:757-810.

3 Liou J, Kim ML, Heo WD, et al. STIM is a Ca2+ sensor essential for $\mathrm{Ca} 2+$-store-depletion-triggered $\mathrm{Ca} 2+$ influx. Curr Biol 2005; 15:1235-1241.

4 Roos J, DiGregorio PJ, Yeromin AV, et al. STIM1, an essential and conserved component of store-operated $\mathrm{Ca} 2+$ channel function. J Cell Biol 2005; 169:435-445.

5 Feske S, Gwack Y, Prakriya M, et al. A mutation in Orail causes immune deficiency by abrogating CRAC channel function. Nature 2006; 441:179-185.

6 Zhang SL, Yeromin AV, Zhang XH, et al. Genome-wide RNAi screen of $\mathrm{Ca}(2+)$ influx identifies genes that regulate $\mathrm{Ca}(2+)$ release-activated $\mathrm{Ca}(2+)$ channel activity. Proc Natl Acad Sci USA 2006; 103:9357-9362.

7 Vig M, Peinelt C, Beck A, et al. CRACM1 is a plasma membrane protein essential for store-operated $\mathrm{Ca} 2+$ entry. Science 2006; 312:1220-1223.

8 Zhang SL, Yu Y, Roos J, et al. STIM1 is a Ca2+ sensor that activates CRAC channels and migrates from the $\mathrm{Ca} 2+$ store to the plasma membrane. Nature 2005; 437:902-905.

9 Prakriya M, Feske S, Gwack Y, Srikanth S, Rao A, Hogan PG. Orail is an essential pore subunit of the CRAC channel. Nature 2006; 443:230-233.

10 Vig M, Beck A, Billingsley JM, et al. CRACM1 multimers form the ion-selective pore of the CRAC channel. Curr Biol 2006; 16:2073-2079.

11 Yeromin AV, Zhang SL, Jiang W, Yu Y, Safrina O, Cahalan MD. Molecular identification of the CRAC channel by altered ion selectivity in a mutant of Orai. Nature 2006; 443:226-229.

$12 \mathrm{Xu} \mathrm{P,} \mathrm{Lu} \mathrm{J,} \mathrm{Li} \mathrm{Z,} \mathrm{Yu} \mathrm{X,} \mathrm{Chen} \mathrm{L,} \mathrm{Xu} \mathrm{T.} \mathrm{Aggregation} \mathrm{of} \mathrm{STIM1}$ underneath the plasma membrane induces clustering of Orai1. Biochem Biophys Res Commun 2006; 350:969-976.

13 Luik RM, Wu MM, Buchanan J, Lewis RS. The elementary unit of store-operated $\mathrm{Ca} 2+$ entry: local activation of CRAC channels by STIM1 at ER-plasma membrane junctions. J Cell Biol 2006; 174:815-825.

14 Mercer JC, Dehaven WI, Smyth JT, et al. Large store-operated calcium selective currents due to co-expression of Orai1 or Orai2 with the intracellular calcium sensor, Stim1. J Biol Chem 2006; 281:24979-24990.

15 Park CY, Hoover PJ, Mullins FM, et al. STIM1 clusters and activates CRAC channels via direct binding of a cytosolic domain to Orai1. Cell 2009; 136:876-890.

16 Zhou Y, Meraner P, Kwon HT, et al. STIM1 gates the storeoperated calcium channel ORAI1 in vitro. Nat Struct Mol Biol 2010; 17:112-116.

17 Ji W, Xu P, Li Z, et al. Functional stoichiometry of the unitary calcium-release-activated calcium channel. Proc Natl Acad Sci USA 2008; 105:13668-13673.

18 Mignen O, Thompson JL, Shuttleworth TJ. Orail subunit stoichiometry of the mammalian CRAC channel pore. J Physiol 2008; 586:419-425.

19 Penna A, Demuro A, Yeromin AV, et al. The CRAC channel consists of a tetramer formed by Stim-induced dimerization of Orai dimers. Nature 2008; 456:116-120.
20 Maruyama Y, Ogura T, Mio K, et al. Tetrameric Orail is a teardrop-shaped molecule with a long, tapered cytoplasmic domain. J Biol Chem 2009; 284:13676-13685.

21 Luik RM, Wang B, Prakriya M, Wu MM, Lewis RS. Oligomerization of STIM1 couples ER calcium depletion to CRAC channel activation. Nature 2008; 454:538-542.

22 Hogan PG, Lewis RS, Rao A. Molecular basis of calcium signaling in lymphocytes: STIM and ORAI. Annu Rev Immunol 2010; 28:491-533.

23 Yuan JP, Zeng W, Dorwart MR, Choi YJ, Worley PF, Muallem S. SOAR and the polybasic STIM1 domains gate and regulate Orai channels. Nat Cell Biol 2009; 11:337-343.

24 Mori MX, Erickson MG, Yue DT. Functional stoichiometry and local enrichment of calmodulin interacting with $\mathrm{Ca} 2+$ channels. Science 2004; 304:432-435.

25 Li Z, Lu J, Xu P, Xie X, Chen L, Xu T. Mapping the interacting domains of STIM1 and Orail in $\mathrm{Ca} 2+$ release-activated Ca2+ channel activation. J Biol Chem, 2007; 282:2944829456.

26 Soboloff J, Spassova MA, Tang XD, Hewavitharana T, Xu W, Gill DL. Orail and STIM reconstitute store-operated calcium channel function. J Biol Chem 2006; 281:20661-20665.

27 Muik M, Frischauf I, Derler I, et al. Dynamic coupling of the putative coiled-coil domain of ORAI1 with STIM1 mediates ORAI1 channel activation. J Biol Chem 2008; 283:80148022.

28 Zhou Y, Meraner P, Kwon HT, et al. STIM1 gates the storeoperated calcium channel ORAI1 in vitro. Nat Struct Mol Biol 2010; 17:112-116.

29 Muik M, Fahrner M, Derler I, et al. A Cytosolic homomerization and a modulatory domain within STIM1 C terminus determine coupling to ORAI1 channels. J Biol Chem 2009; 284:8421-8426.

30 Peinelt C, Vig M, Koomoa DL, et al. Amplification of CRAC current by STIM1 and CRACM1 (Orai1). Nat Cell Biol 2006; 8:771-773.

31 Zheng J, Sigworth FJ. Intermediate conductances during deactivation of heteromultimeric Shaker potassium channels. J Gen Physiol 1998; 112:457-474.

32 Rosenmund C, Stern-Bach Y, Stevens CF. The tetrameric structure of a glutamate receptor channel. Science 1998; 280:1596-1599.

33 Sobolevsky AI, Rosconi MP, Gouaux E. X-ray structure, symmetry and mechanism of an AMPA-subtype glutamate receptor. Nature 2009; 462:745-756.

34 Ruiz ML, Karpen JW. Single cyclic nucleotide-gated channels locked in different ligand-bound states. Nature 1997; 389:389-392.

35 Dolmetsch RE, Lewis RS, Goodnow CC, Healy Ji. Differential activation of transcription factors induced by $\mathrm{Ca} 2+$ response amplitude and duration. Nature 1997; 386:855-858.

36 Dolmetsch RE, Xu K, Lewis RS. Calcium oscillations increase the efficiency and specificity of gene expression. $\mathrm{Na}$ ture 1998. 392:933-936.

37 Chen Y, Deng Y, Zhang J, Yang L, Xie X, Xu T. GDI-1 preferably interacts with Rab10 in insulin-stimulated GLUT4 translocation. Biochem J 2009; 422:229-235.

(Supplementary information is linked to the online version of the paper on the Cell Research website.) 